PROCEEDINGS OF THE

AMERICAN MATHEMATICAL SOCIETY

Volume 139, Number 1, January 2011, Pages 209-217

S 0002-9939(2010)10488-4

Article electronically published on July 7, 2010

\title{
A SCHWARZ-PICK THEOREM FOR HIGHER-ORDER HYPERBOLIC DERIVATIVES
}

\author{
PATRICE RIVARD
}

(Communicated by Mario Bonk)

\begin{abstract}
Using the hyperbolic divided differences introduced by Baribeau, Rivard and Wegert, we generalize the notion of hyperbolic derivative and we give the analogue of a Schwarz-Pick Theorem for these derivatives.
\end{abstract}

\section{INTRODUCTION}

In 1, we introduced the concept of hyperbolic divided differences and showed that they provide a natural analogue of classical Newton interpolation in the context of interpolation in the unit disk. In this paper, we show how hyperbolic divided differences can be used, in a natural way, to define a notion of higher-order hyperbolic derivatives.

We will begin by recalling some essential elements from this theory. Throughout the text, we will denote by $\mathbb{D}$ the unit disk and by $\mathbb{T}$ the unit circle. We also use the notation

$$
\overline{\mathbb{D}}:=\mathbb{D} \cup \mathbb{T}, \quad \widehat{\mathbb{D}}:=\overline{\mathbb{D}} \cup\{\infty\} .
$$

Let $z_{1}, z_{2}$ be points of $\mathbb{D}$. The hyperbolic distance $\rho$ between the points $z_{1}$ and $z_{2}$ is given by

$$
\rho\left(z_{1}, z_{2}\right)=\log \left(\frac{1+p\left(z_{1}, z_{2}\right)}{1-p\left(z_{1}, z_{2}\right)}\right), \quad p\left(z_{1}, z_{2}\right):=\left|\frac{z_{1}-z_{2}}{1-\overline{z_{2}} z_{1}}\right| .
$$

The quantity $p\left(z_{1}, z_{2}\right)$ is called the pseudo-hyperbolic distance of the points $z_{1}, z_{2}$.

In the case where at least one of the two points belongs to $\mathbb{T}$, we set

$$
\rho\left(z_{1}, z_{2}\right):=\left\{\begin{array}{cc}
\infty & \text { if } z_{1} \neq z_{2} \\
0 & \text { if } z_{1}=z_{2}
\end{array}\right.
$$

Definition 1.1. Let $z_{1}, z_{2} \in \widehat{\mathbb{D}}$. We define $\left[z_{1}, z_{2}\right]$ by

$$
\left[z_{1}, z_{2}\right]:=\left\{\begin{array}{cc}
\frac{z_{2}-z_{1}}{1-\overline{z_{2}} z_{1}} & \text { if } z_{1}, z_{2} \in \overline{\mathbb{D}}, \overline{z_{2}} \cdot z_{1} \neq 1 \\
z_{2} & \text { if } z_{1}=z_{2} \in \mathbb{T} \\
\infty & \text { otherwise }
\end{array}\right.
$$

Received by the editors August 19, 2009 and, in revised form, February 24, 2010.

2010 Mathematics Subject Classification. Primary 30F45; Secondary 30C80.

Key words and phrases. Schwarz-Pick Theorem, hyperbolic derivative, hyperbolic divided differences.

The author was supported by the NSERC Postgraduate Scholarships program.

(C)2010 American Mathematical Society Reverts to public domain 28 years from publication 
Remark 1.2. At first glance, Definition 1.1 seems strange in the case where $z_{1}=$ $z_{2} \in \mathbb{T}$, but it finds its meaning in [1]. For the sake of consistency, we have decided to retain this definition.

One checks easily the following identities, which will be used later:

$$
\begin{aligned}
{[[\zeta, z], z] } & =\zeta, \quad \zeta \in \widehat{\mathbb{D}}, z \in \mathbb{D}, \\
{\left[e^{i \theta} z_{1}, e^{i \theta} z_{2}\right] } & =e^{i \theta}\left[z_{1}, z_{2}\right], \quad z_{1}, z_{2} \in \widehat{\mathbb{D}}, \theta \in \mathbb{R} .
\end{aligned}
$$

In the following we will denote by $\mathcal{H}$ the class of analytic functions of the unit disk $\mathbb{D}$ into itself,

$$
\mathcal{H}:=\{f: f \text { holomorphic in } \mathbb{D},|f(z)|<1, z \in \mathbb{D}\},
$$

and we shall consider also $\mathcal{S}$, the $S$ chur class,

$$
\mathcal{S}:=\{f: f \text { holomorphic in } \mathbb{D},|f(z)| \leq 1, z \in \mathbb{D}\} .
$$

A Blaschke product of degree $n \geq 1$ is a function of the form

$$
B(z):=e^{i \theta} \prod_{j=1}^{n} \frac{z-z_{j}}{1-\overline{z_{j}} z}, \quad\left|z_{j}\right|<1, \theta \in \mathbb{R} .
$$

The unimodular constants are considered to be Blaschke products of degree 0. For $n \geq 0$, we will denote the class of Blaschke products of degree $n$ by $\mathcal{B}_{n}$.

We are now prepared to give the definition of the hyperbolic divided differences.

Definition 1.3. Let $f \in \mathcal{S}$ and fix $k$ pairwise distinct points $z_{1}, \ldots, z_{k} \in \mathbb{D}$. Set

$$
\Delta^{0} f(z):=f(z), \quad z \in \mathbb{D} .
$$

We define recursively for $j=1, \ldots, k$ the hyperbolic divided difference of order $j$ of the function $f$ with parameters $z_{1}, \ldots, z_{j}$, denoted $\Delta^{j} f\left(z ; z_{1}, \ldots, z_{j}\right)$, as follows:

$$
\Delta^{j} f\left(z ; z_{1}, \ldots, z_{j}\right):=\frac{\left[\Delta^{j-1} f\left(z ; z_{1}, \ldots, z_{j-1}\right), \Delta^{j-1} f\left(z_{j} ; z_{1}, \ldots, z_{j-1}\right)\right]}{\left[z, z_{j}\right]},
$$

provided $\Delta^{j-1} f\left(z ; z_{1}, \ldots, z_{j-1}\right) \notin \mathcal{B}_{0}$. The quotient in the right-hand side of (1.4) has to be interpreted as a limit if $z=z_{j}$.

If $\Delta^{j-1} f\left(z ; z_{1}, \ldots, z_{j-1}\right) \in \mathcal{B}_{0}$, we define

$$
\Delta^{j} f\left(z ; z_{1}, \ldots, z_{j}\right):=\Delta^{j-1} f\left(z_{j} ; z_{1}, \ldots, z_{j-1}\right) .
$$

We also introduce the operators $\Delta_{z_{1}, \ldots, z_{j}}^{j}$, which act on $f$ in $\mathcal{S}$ as follows:

$$
\Delta_{z_{1}, \ldots, z_{j}}^{j} f(z):=\Delta^{j} f\left(z ; z_{1}, \ldots, z_{j}\right), \quad z \in \mathbb{D}, j=1, \ldots, k .
$$

This allows us to rewrite the above recursive definition of $\Delta^{j} f\left(z ; z_{1}, \ldots, z_{j}\right)$ as

$$
\Delta^{j} f\left(z ; z_{1}, \ldots, z_{j}\right)=\left(\Delta_{z_{j}}^{1}\left(\Delta_{z_{1}, \ldots, z_{j-1}}^{j-1} f\right)\right)(z), \quad j=1, \ldots, k .
$$

It was shown in [1] that $\Delta_{z_{1}, \ldots, z_{j}}^{j} f$ is well-defined and belongs to $\mathcal{S}$ for all $f \in \mathcal{S}$. The fact that we adopt the convention (1.5) is a judicious choice in order to solve interpolation problems [1].

The hyperbolic divided differences have the property that they act in a particular way on the class of Blaschke products. This is the next assertion. 
Theorem 1.4 ([1]). Let $z_{1}, \ldots, z_{k} \in \mathbb{D}$ be pairwise distinct points and let the operator $\Delta^{k}: \mathcal{S} \rightarrow \mathcal{S}$ be defined by

$$
\Delta^{k} f(z):=\Delta^{k} f\left(z ; z_{1}, \ldots, z_{k}\right), \quad z \in \mathbb{D} .
$$

Then $f \in \mathcal{S}$ is a Blaschke product of degree $n>k$ if and only if $\Delta^{k} f$ is a Blaschke product of degree $n-k$ and $f$ is a Blaschke product of degree $n \leq k$ if and only if $\Delta^{k} f$ is a Blaschke product of degree zero.

The Schwarz-Pick Theorem together with Theorem 1.4 leads to the following "multi-point Schwarz-Pick Theorem".

Theorem 1.5 ([1]). Let $z_{1}, \ldots, z_{k} \in \mathbb{D}$ be pairwise distinct points. Then, for all $f \in \mathcal{S}$ and for $u, v \in \mathbb{D}$,

$$
\rho\left(\Delta^{k} f\left(u ; z_{1}, \ldots, z_{k}\right), \Delta^{k} f\left(v ; z_{1}, \ldots, z_{k}\right)\right) \leq \rho(u, v) .
$$

If equality holds in (1.6) for a pair of distinct points $u$ and $v$, then $f$ is a Blaschke product of degree $k+1$ and equality holds for all $u, v \in \mathbb{D}$.

\section{Hyperbolic Divided DIFFERENCES WITH ONE PARAMETER}

In defining hyperbolic divided differences, we have assumed that the parameters are pairwise distinct points in the unit disk. For our purpose, we shall deal, in this section, with a hyperbolic divided difference with a single parameter.

Definition 2.1. Let $f \in \mathcal{S}, z \in \mathbb{D}$ and $n \geq 1$. For $\zeta \in \mathbb{D}$, we define the hyperbolic divided difference of order $n$ of $f$ with parameter $z$, denoted $\Delta_{z}^{n} f(\zeta)$, by

$$
\Delta_{z}^{n} f(\zeta) \equiv \Delta^{n} f(\zeta ; z, \ldots, z):=\lim _{z_{n} \rightarrow z z_{n-1} \rightarrow z} \lim _{z_{1} \rightarrow z} \lim ^{n} f\left(\zeta ; z_{1}, \ldots, z_{n}\right) \quad(\zeta \neq z)
$$

and

$$
\Delta_{z}^{n} f(z)=\Delta^{n} f(z ; z, \ldots, z):=\lim _{\zeta \rightarrow z} \Delta^{n} f(\zeta ; z, \ldots, z) .
$$

Remark 2.2. Formally, we have

$$
\Delta_{z}^{n}(f)(\zeta)=\lim _{z_{n} \rightarrow z} \lim _{z_{n-1} \rightarrow z} \ldots \lim _{z_{1} \rightarrow z} \Delta_{z_{n}}^{1}\left(\Delta_{z_{1}, \ldots, z_{n-1}}^{n-1} f\right)(\zeta) .
$$

For brevity, we simply write

$$
\Delta_{z}^{n} f(\zeta)=\left(\Delta_{z}^{1}\left(\Delta_{z}^{n-1} f\right)\right)(\zeta)
$$

rather than (2.3).

It remains to show that definitions (2.1) and (2.2) make sense. To do this, we will prove by induction that $\Delta_{z}^{n} f \in \mathcal{S}$ for $f \in \mathcal{S}$ and $z \in \mathbb{D}$. The case $n=1$ just follows from Lemma 2.2 [1]. Now assume that $\Delta_{z}^{n} f \in \mathcal{S}$ if $f \in \mathcal{S}$. Using the operator notation and continuity, we get for $n+1$,

$$
\begin{aligned}
\Delta_{z}^{n+1} f(\zeta) & =\lim _{z_{n+1} \rightarrow z} \lim _{z_{n} \rightarrow z} \ldots \lim _{z_{1} \rightarrow z} \Delta^{n+1} f\left(\zeta ; z_{1}, \ldots, z_{n+1}\right) \\
& =\lim _{z_{n+1} \rightarrow z} \lim _{z_{n} \rightarrow z} \ldots \lim _{z_{1} \rightarrow z} \Delta_{z_{n+1}}^{1}\left(\Delta_{z_{1}, \ldots, z_{n}}^{n} f\right)(\zeta) \\
& =\Delta_{z}^{1}\left(\Delta_{z}^{n} f(\zeta)\right) .
\end{aligned}
$$

We deduce by the case $n=1$ and the induction hypothesis that $\Delta_{z}^{n+1} f \in \mathcal{S}$. This implies that the definitions make sense, as required. We have proved the next assertion. 
Proposition 2.3. Let $f \in \mathcal{S}, z \in \mathbb{D}$ and $n \geq 1$. Then $\Delta_{z}^{n} f \in \mathcal{S}$.

Theorem 1.4 and Theorem 1.5 are still valid if we replace the pairwise distinct parameters by a single one.

Theorem 2.4. Let $z \in \mathbb{D}$ and let the operator $\Delta^{k}: \mathcal{S} \rightarrow \mathcal{S}$ be defined by

$$
\Delta^{k} f(\zeta):=\Delta_{z}^{k} f(\zeta), \quad \zeta \in \mathbb{D} .
$$

Then $f \in \mathcal{S}$ is a Blaschke product of degree $n>k$ if and only if $\Delta^{k} f$ is a Blaschke product of degree $n-k$ and $f$ is a Blaschke product of degree $n \leq k$ if and only if $\Delta^{k} f$ is a Blaschke product of degree zero.

Proof. We use induction on $k$. For $k=1$, the result follows from Theorem 1.4 Suppose the result is true for $k$. Then, by Remark 2.2, we can write

$$
\Delta_{z}^{k+1} f(\zeta)=\left(\Delta_{z}^{1}\left(\Delta_{z}^{k} f\right)\right)(\zeta)
$$

and the result for $k+1$ follows from (2.4), the case $k=1$, Proposition 2.3 and the induction hypothesis.

Theorem 2.5. Let $z \in \mathbb{D}$. Then, for all $f \in \mathcal{S}$ and for $u, v \in \mathbb{D}$,

$$
\rho\left(\Delta_{z}^{k} f(u), \Delta_{z}^{k} f(v)\right) \leq \rho(u, v) .
$$

If equality holds in (2.5) for a pair of distinct points $u$ and $v$, then $f$ is a Blaschke product of degree $k+1$ and equality holds for all $u, v \in \mathbb{D}$.

Proof. Proposition 2.3 implies that $\Delta_{z}^{k} f \in \mathcal{S}$. If $\Delta_{z}^{k} f \in \mathcal{H}$, we can deduce (2.5) from the classical Schwarz-Pick Theorem. If $\Delta_{z}^{k} f \in \mathcal{B}_{0}$, the left-hand side of (2.5) is zero, while the right-hand side is positive if $u \neq v$. Finally, the case of equality is a consequence of Theorem 2.4 and the case of equality in the classical Schwarz-Pick Theorem.

\section{HigheR-ORDER HYPERBOLIC DERIVATIVES}

The notion of a hyperbolic derivative is well known ([3], 4] and [2]). Indeed, for $f \in \mathcal{H}$, the hyperbolic derivative of $f$ is defined as

$$
f^{h}(z):=\frac{f^{\prime}(z)\left(1-|z|^{2}\right)}{1-|f(z)|^{2}} \quad(z \in \mathbb{D}),
$$

which coincides with $\Delta_{z}^{1} f(z)$. In accordance with our earlier conventions, in the case where $f$ is a Blaschke product of degree zero, we define the hyperbolic derivative by

$$
f^{h}(z):=f(z) .
$$

The hyperbolic divided differences suggest a possible extension of the notion of hyperbolic derivative to higher orders. Indeed, it seems natural to define the higher-order hyperbolic derivatives by using the higher-order hyperbolic divided differences with one parameter. This brings us to the following definition.

Definition 3.1. Let $f \in \mathcal{S}$ and $n \geq 1$. The hyperbolic derivative of order $n$ of $f$ at the point $z \in \mathbb{D}$, denoted $H^{n} f(z)$, is defined by

$$
H^{n} f(z):=\Delta_{z}^{n} f(z) .
$$


Recall that by Proposition 2.3 this definition makes sense.

Suppose that $f \in \mathcal{H}$. To compute $H^{n} f(z)$, we need to introduce the following. For $z \in \mathbb{D}$, we define $D^{n} f(z)$ by the Taylor series expansion of the function

$$
\zeta \mapsto g(\zeta):=-[f([-\zeta, z]), f(z)]=\frac{f\left(\frac{z+\zeta}{1+\bar{z} \zeta}\right)-f(z)}{1-\overline{f(z)} f\left(\frac{z+\zeta}{1+\bar{z} \zeta}\right)}=\sum_{n=1}^{\infty} \frac{D^{n} f(z)}{n !} \zeta^{n},
$$

where

$$
D^{1} f(z):=g^{\prime}(0), D^{2} f(z):=g^{\prime \prime}(0), \ldots
$$

For example,

$$
\begin{aligned}
& D^{1} f(z)=\frac{\left(1-|z|^{2}\right) f^{\prime}(z)}{1-|f(z)|^{2}} \\
& D^{2} f(z)=\frac{f^{\prime \prime}(z)\left(1-|z|^{2}\right)^{2}}{1-|f(z)|^{2}}-\frac{2 \bar{z} f^{\prime}(z)\left(1-|z|^{2}\right)}{1-|f(z)|^{2}}+\frac{2 \overline{f(z)} f^{\prime}(z)^{2}\left(1-|z|^{2}\right)^{2}}{\left(1-|f(z)|^{2}\right)^{2}} .
\end{aligned}
$$

These derivatives $D^{n} f$ were first introduced by Peschl [7] and are usually referred to as differential invariants because

$$
\left|D^{n}(\psi \circ f \circ \phi)(z)\right|=\left|D^{n}(f)(\phi(z))\right|
$$

whenever $\psi$ and $\phi$ are automorphisms of the unit disk [5].

The next theorem is easily proved using the definition and some calculations.

Theorem 3.2. For a given $z \in \mathbb{D}$, we have the following identities:

(1) if $f \in \mathcal{H}$, then

(2) if $f \in \mathcal{H} \backslash \mathcal{B}_{1}$, then

$$
H^{1} f(z)=D^{1} f(z)
$$

$$
H^{2} f(z)=\frac{1}{2} \frac{1}{1-\left|D^{1} f(z)\right|^{2}} D^{2} f(z) .
$$

The hyperbolic derivatives $H^{n} f(z)$ are invariant in the same sense as the differential invariants $D^{n} f(z)$. But before establishing a relation equivalent to (3.2), we need the next lemma.

Lemma 3.3. Let $\psi, \phi$ be automorphisms of the unit disk and let $f \in \mathcal{S}$. For $n \geq 1$, suppose that $z_{1}, \ldots, z_{n} \in \mathbb{D}$ are pairwise distinct points. Then for $1 \leq j \leq n$, there exists $\theta \in \mathbb{R}$ such that

$$
\Delta^{j}(\psi \circ f \circ \phi)\left(z ; z_{1}, \ldots, z_{j}\right)=e^{i \theta} \Delta^{j} f\left(\phi(z) ; \phi\left(z_{1}\right), \ldots, \phi\left(z_{j}\right)\right), z \in \mathbb{D} .
$$

Proof. If $f \in \mathcal{B}_{0}$, the result is clear. Suppose now that $f \in \mathcal{H}$. We have

$$
\begin{aligned}
\Delta^{1}(f \circ \phi)\left(z ; z_{1}\right) & =\frac{\left[(f \circ \phi)(z),(f \circ \phi)\left(z_{1}\right)\right]}{\left[z, z_{1}\right]} \\
& =\frac{\left[f(\phi(z)), f\left(\phi\left(z_{1}\right)\right)\right]}{\left[\phi(z), \phi\left(z_{1}\right)\right]} \frac{\left[\phi(z), \phi\left(z_{1}\right)\right]}{\left[z, z_{1}\right]} \\
& =e^{i \theta} \Delta^{1} f\left(\phi(z) ; \phi\left(z_{1}\right)\right)
\end{aligned}
$$

for some $\theta \in \mathbb{R}$, by Theorem 1.4. Using identity (1.3), it then follows by induction that for each $j \geq 1$,

$$
\Delta^{j}(f \circ \phi)\left(z ; z_{1}, \ldots, z_{j}\right)=e^{i \theta} \Delta^{j} f\left(\phi(z) ; \phi\left(z_{1}\right), \ldots, \phi\left(z_{j}\right)\right), \quad z \in \mathbb{D} .
$$


Similarly, Theorem 1.4 gives

$$
\begin{aligned}
\Delta^{1}(\psi \circ f)\left(z ; z_{1}\right) & =\frac{\left[(\psi \circ f)(z),(\psi \circ f)\left(z_{1}\right)\right]}{\left[z, z_{1}\right]} \\
& =\frac{\left[\psi(f(z)), \psi\left(f\left(z_{1}\right)\right)\right]}{\left[f(z), f\left(z_{1}\right)\right]} \frac{\left[f(z), f\left(z_{1}\right)\right]}{\left[z, z_{1}\right]} \\
& =e^{i \tilde{\theta}} \Delta^{1} f\left(z ; z_{1}\right),
\end{aligned}
$$

for some $\tilde{\theta} \in \mathbb{R}$, and again by induction we get for $j \geq 1$ that

$$
\Delta^{j}(\psi \circ f)\left(z ; z_{1}, \ldots, z_{j}\right)=e^{i \tilde{\theta}} \Delta^{j} f\left(z ; z_{1}, \ldots, z_{j}\right), \quad z \in \mathbb{D} .
$$

Finally, by combining (3.4) and (3.5), we obtain (3.3), as required.

The invariance of the higher-order hyperbolic derivatives follows directly from Lemma 3.3. This is the next theorem.

Theorem 3.4. Suppose that $\psi, \phi$ are automorphisms of the unit disk, $f \in \mathcal{S}$ and $n \geq 1$. Then

$$
\left|H^{n}(\psi \circ f \circ \phi)(z)\right|=\left|H^{n}(f)(\phi(z))\right|, \quad z \in \mathbb{D} .
$$

In the same way that the value of a polynomial and that of its first $n$ derivatives at a point determine entirely a polynomial of degree $n$, a Blaschke product of degree $n$ is entirely determined by its value and that of its first $n$ hyperbolic derivatives at any given point. This is the content of the next result, where we give a procedure for constructing the Blaschke product. We essentially combine the concept presented here with an algorithm given by Baribeau, Rivard and Wegert for solving interpolation problems ([1], Theorem 4.1).

Theorem 3.5. Let $z_{0} \in \mathbb{D}$ and $n \geq 1$. Fix $n+1$ points $\omega_{0}, \ldots, \omega_{n} \in \overline{\mathbb{D}}$, where $\left|w_{j}\right|<1$, for $j=0, \ldots, n-1$ and $\left|\omega_{n}\right|=1$. Then there exists a unique Blaschke product $b$ of degree $n$ such that

$$
H^{j} b\left(z_{0}\right)=\omega_{j}, \quad j=0, \ldots, n,
$$

with the convention that $H^{0} b\left(z_{0}\right)=b\left(z_{0}\right)$.

Proof. Starting with $b_{0}:=\omega_{n}$ we define the functions $b_{1}, \ldots, b_{n}$ recursively by

$$
b_{k+1}(z):=\left[\left[z, z_{0}\right] \cdot b_{k}(z), \omega_{n-k-1}\right], \quad k=0, \ldots, n-1 .
$$

First, it is immediate from this definition that $b_{k+1}\left(z_{0}\right)=w_{n-k-1}$, for $k=0, \ldots$, $n-1$. Together with (1.2) and the definition, this gives $\Delta_{z_{0}}^{1} b_{k+1}(z)=b_{k}(z)$, for $k=0, \ldots, n-1$, and, by induction, $\Delta_{z_{0}}^{j} b_{n}(z)=b_{n-j}(z)$, for $j=1, \ldots, n$. In particular, by Theorem 2.4, $b_{n}$ is a Blaschke product which satisfies (3.6). Finally, in view of (1.2), this construction is unique.

\section{A Schwarz-Pick Theorem For hyperbolic Derivatives}

With the notion of higher-order hyperbolic derivatives available, we are able to give a version of the Schwarz-Pick Theorem for them. We note an analogue of a Schwarz-Pick Theorem for hyperbolic derivatives due to Beardon [3]. We can also find it in [4] and [2]. 
Theorem 4.1 ([3]). Let $f \in \mathcal{H} \backslash \mathcal{B}_{1}$ and suppose that $f(0)=0$. Then

$$
\rho\left(f^{h}(0), f^{h}(z)\right) \leq 2 \rho(0, z), \quad z \in \mathbb{D} .
$$

Further, equality holds in (4.1) for $f(z)=z^{2}$.

The next assertion is the main result of this paper. On the one hand, it extends Theorem 4.1 to hyperbolic derivatives of order greater than one and on the other hand, it gives sufficient and necessary conditions concerning the case of equality. The proof is relatively simple and is modelled on the proofs of Corollary 3.6 and Theorem 5.4 given respectively in [4] and [2].

Theorem 4.2. Let $f \in \mathcal{S}, u, v \in \mathbb{D}$ and $n \geq 1$. Then

$$
\rho\left(H^{n} f(u), H^{n} f(v)\right)-\rho\left(\Delta_{u}^{n} f(v), \Delta_{v}^{n} f(u)\right) \leq 2 \rho(u, v) .
$$

Further, equality holds in (4.2) for a pair of distinct points $u$ and $v$ if and only if $f$ is a Blaschke product of degree $n+1$ and also if $u, v,\left(\Delta_{u}^{n} f\right)^{-1}\left(\Delta_{v}^{n} f(u)\right)$ and $\left(\Delta_{u}^{n} f\right)^{-1}\left(H^{n} f(v)\right)$ belong, in this order, to the same hyperbolic geodesic.

Remark 4.3. If $f$ is a Blaschke product of degree $n+1$, then, by Theorem 2.4] it follows that $\Delta_{u}^{n} f$ and $\Delta_{v}^{n} f$ are automorphisms of the unit disk. Thus, the inverse function $\left(\Delta_{u}^{n} f\right)^{-1}$ is well defined.

Proof. First, suppose that $f \in \bigcup_{k=0}^{n} \mathcal{B}_{k}$. Then, $H^{n} f(u)=H^{n} f(v)=\lambda,|\lambda|=1$ and $\Delta_{u}^{n} f(v), \Delta_{v}^{n} f(u)$ are unimodular constants. The right-hand side of (4.2) is a positive constant and, using (1.1), we see that the left-hand side is either zero or $-\infty$. With the convention that $-\infty$ is smaller than any real number, we obtain the result.

Next, suppose that $f \in \mathcal{S} \backslash \bigcup_{k=0}^{n} \mathcal{B}_{k}$. Applying the triangle inequality twice, we have

$$
\begin{aligned}
& \rho\left(H^{n} f(u), H^{n} f(v)\right)=\rho\left(\Delta^{n} f(u ; u, \ldots, u), \Delta^{n} f(v ; v, \ldots, v)\right) \\
& \leq \rho\left(\Delta^{n} f(u ; u, \ldots, u), \Delta^{n} f(v ; u, \ldots, u)\right)+\rho\left(\Delta^{n} f(v ; u, \ldots, u), \Delta^{n} f(v ; v, \ldots, v)\right) \\
& \leq \rho\left(\Delta^{n} f(u ; u, \ldots, u), \Delta^{n} f(v ; u, \ldots, u)\right)+\rho\left(\Delta^{n} f(v ; u, \ldots, u), \Delta^{n} f(u ; v, \ldots, v)\right) \\
& +\rho\left(\Delta^{n} f(u ; v, \ldots, v), \Delta^{n} f(v ; v, \ldots, v)\right) \\
& \leq 2 \rho(u, v)+\rho\left(\Delta^{n} f(u ; v, \ldots, v), \Delta^{n} f(v ; u, \ldots, u)\right),
\end{aligned}
$$

where the last inequality follows from Theorem 2.5. Finally, we get

$$
\rho\left(H^{n} f(u), H^{n} f(v)\right) \leq 2 \rho(u, v)+\rho\left(\Delta_{u}^{n} f(v), \Delta_{v}^{n} f(u)\right) .
$$

Suppose now that $f$ is a Blaschke product of degree $n+1$ and that $u, v$, $\left(\Delta_{u}^{n} f\right)^{-1}\left(\Delta_{v}^{n} f(u)\right)$ and $\left(\Delta_{u}^{n} f\right)^{-1}\left(H^{n} f(v)\right)$ belong to the same hyperbolic geodesic. Applying the automorphism $\Delta_{u}^{n} f$, which acts as an isometry in the hyperbolic metric, to these points, we obtain that $\Delta_{u}^{n} f(u)=H^{n} f(u), \Delta_{u}^{n} f(v), \Delta_{v}^{n} f(u)$ and $H^{n} f(v)$ belong to the same hyperbolic geodesic. This implies that the first two inequalities are in fact equalities in the preceding proof. Finally, the last inequality is also an equality as a consequence of Theorem 2.5 .

Conversely, if equality holds in (4.2), then equality holds everywhere in the previous sequence of inequalities. The last equality implies that $f$ is a Blaschke product of degree $n+1$, and the previous two that $H^{n} f(u), \Delta_{u}^{n} f(v), \Delta_{v}^{n} f(u)$ and $H^{n} f(v)$ lie in this order on the same hyperbolic geodesic. Since $\Delta_{u}^{n} f$ is an automorphism of the unit disk, then $u, v,\left(\Delta_{u}^{n} f\right)^{-1}\left(\Delta_{v}^{n} f(u)\right)$, and $\left(\Delta_{u}^{n} f\right)^{-1}\left(H^{n} f(v)\right)$ lie on the same hyperbolic geodesic, in this order. 
We should mention the work of P. R. Mercer, who has obtained a Schwarz-Picktype estimate which improved Theorem 4.1 in [6] and whose result corresponds to the case $n=1$ of Theorem 4.2. He also used techniques quite different from those appearing in the proof of the theorem.

Theorem 4.1 requires the normalization $f(0)=0$, and the conclusion does not hold in general if 0 and $z$ are replaced by $w$ and $z$. However the following result was obtained by Beardon and Minda in [2], 4].

Theorem 4.4 ([2]). Suppose that $f: \mathbb{D} \rightarrow \mathbb{D}$ is holomorphic but not a conformal automorphism of $\mathbb{D}$. Then for all $z, w \in \mathbb{D}$,

$$
\rho\left(\left|f^{h}(z)\right|,\left|f^{h}(w)\right|\right) \leq 2 \rho(z, w) .
$$

A reasonable conjecture is that inequality (4.3) is still valid in the case of hyperbolic derivatives of higher order. But for the hyperbolic derivative of order two, the function $f(z)=z^{3}$, with the pair of points $z_{1}=0, z_{2}=1 / 4$, provides an easy counterexample. Indeed, we have that

$$
\begin{aligned}
\rho\left(\left|H^{2} f(0)\right|,\left|H^{2} f(1 / 4)\right|\right) & =0.7506121639 ; \\
2 \rho(0,1 / 4) & =0.5108256238 .
\end{aligned}
$$

As Theorem 4.6 will show, an additive term is essential to establish a proper upper bound to get an analogous result in the case of hyperbolic derivatives of higher order. But before that, we will need the following simple lemma.

Lemma 4.5. Consider $z, w$ to be a pair of distinct points in $\mathbb{D}$. Then

$$
\rho(|z|,|w|) \leq \rho(z, w) \text {. }
$$

Further, equality holds in (4.4) if and only if $z$ and $w$ lie on a hyperbolic geodesic ray emanating from 0 .

Proof. Suppose, without loss of generality, that $|w|>|z|$. Then

$$
\begin{aligned}
\rho(|z|,|w|) & =\rho(0,|w|)-\rho(0,|z|) \\
& =\rho(0, w)-\rho(0, z) \\
& \leq \rho(z, w) .
\end{aligned}
$$

Suppose now that $z, w$ lie on a hyperbolic geodesic ray emanating from 0 . It is clear that equality holds in (4.4) in that case. Conversely, if equality holds in (4.4), the previous inequality is in fact an equality. This implies that $z, w$ lie on a hyperbolic geodesic ray emanating from 0 .

Theorem 4.6. Let $f \in \mathcal{S}, u, v \in \mathbb{D}$ and $n \geq 1$. Then,

$$
\rho\left(\left|H^{n} f(u)\right|,\left|H^{n} f(v)\right|\right) \leq 2 \rho(u, v)+\rho\left(\left|\Delta_{u}^{n} f(v)\right|,\left|\Delta_{v}^{n} f(u)\right|\right) .
$$

Further, equality holds in (4.5) for a pair of distinct points $u$ and $v$ if and only if $f$ is a Blaschke product of degree $n+1$ and if $H^{n} f(u), \Delta_{u}^{n} f(v), \Delta_{v}^{n} f(u), H^{n} f(v)$ lie in this order on a hyperbolic geodesic ray emanating from 0 .

Proof. First, suppose that $f \in \bigcup_{k=0}^{n} \mathcal{B}_{k}$. Then, $\left|H^{n} f(u)\right|=\left|H^{n} f(v)\right|=\left|\Delta_{u}^{n} f(v)\right|=$ $\left|\Delta_{v}^{n} f(u)\right|=1$. Using (1.1), the right-hand side of (4.5) is a positive constant and the left-hand side is zero, which proves the result in that case. Next, suppose 
that $f \in \mathcal{S} \backslash \bigcup_{k=0}^{n} \mathcal{B}_{k}$. Applying the triangle inequality, using Theorem 2.5 and Lemma 4.5 we have

$$
\begin{aligned}
\rho\left(\left|H^{n} f(u)\right|,\left|H^{n} f(v)\right|\right) & \leq \rho\left(\left|H^{n} f(u)\right|,\left|\Delta_{u}^{n} f(v)\right|\right)+\rho\left(\left|\Delta_{u}^{n} f(v)\right|,\left|H^{n} f(v)\right|\right) \\
& \leq \rho\left(H^{n} f(u), \Delta_{u}^{n} f(v)\right)+\rho\left(\left|\Delta_{u}^{n} f(v)\right|,\left|H^{n} f(v)\right|\right) \\
& \leq \rho(u, v)+\rho\left(\left|\Delta_{u}^{n} f(v)\right|,\left|H^{n} f(v)\right|\right) \\
& \leq \rho(u, v)+\rho\left(\left|\Delta_{u}^{n} f(v)\right|,\left|\Delta_{v}^{n} f(u)\right|\right)+\rho\left(\left|\Delta_{v}^{n} f(u)\right|,\left|H^{n} f(v)\right|\right) \\
& \leq \rho(u, v)+\rho\left(\left|\Delta_{u}^{n} f(v)\right|,\left|\Delta_{v}^{n} f(u)\right|\right)+\rho\left(\Delta_{v}^{n} f(u), H^{n} f(v)\right) \\
& \leq 2 \rho(u, v)+\rho\left(\left|\Delta_{u}^{n} f(v)\right|,\left|\Delta_{v}^{n} f(u)\right|\right) .
\end{aligned}
$$

Suppose now that $f$ is a Blaschke product of degree $n+1$ and that $H^{n} f(u)$, $\Delta_{u}^{n} f(v), \Delta_{v}^{n} f(u), H^{n} f(v)$ lie in this order on a hyperbolic geodesic ray emanating from 0 . Then equality holds in each of the above inequalities. Conversely, if equality holds in (4.5), the result follows easily by the case of equality in Theorem 2.5 and Lemma 4.5

We conclude by asking the following question: is it possible to estimate the last term in (4.5) so as to obtain a positive constant $\alpha$ such that

$$
\rho\left(\left|H^{n} f(u)\right|,\left|H^{n} f(v)\right|\right) \leq \alpha \rho(u, v) ?
$$

This would be more in the spirit of Theorem 4.4 .

\section{ACKNOWLEDGEMENT}

The author wishes to sincerely thank his doctoral thesis advisor, Professor Line Baribeau, for her many valuable suggestions and comments which have greatly improved the present paper.

\section{REFERENCES}

[1] L. Baribeau, P. Rivard, and E. Wegert, On hyperbolic divided differences and the NevanlinnaPick problem, Comput. Methods Funct. Theory 9 (2009), no. 2, 391-405. MR2572646

[2] A. F. Beardon and D. Minda, The hyperbolic metric and geometric function theory, Quasiconformal mappings and their applications, 2007, pp. 9-56. MR.2492498

[3] A.F. Beardon, The Schwarz-Pick Lemma for derivatives, Proc. Amer. Math. Soc. 125 (1997), 3255-3256. MR1401727 (97m:30062)

[4] A.F. Beardon and D. Minda, A multi-point Schwarz-Pick Lemma, J. Anal. Math. 92 (2004), 81-104. MR.2072742 (2005f:30044)

[5] S.-A Kim and T. Sugawa, Invariant differential operators associated with a conformal metric, Michigan Math. J. 55 (2007), 459-479. MR2369945 (2008k:30053)

[6] Peter R. Mercer, Schwarz-Pick-type estimates for the hyperbolic derivative, J. Inequal. Appl. (2006), Art. ID 96368, 6 pp. MR2221232 (2007d:30011)

[7] E. Peschl, Les invariants différentiels non holomorphes et leur rôle dans la théorie des fonctions, Rend. Sem. Mat. Messina Ser. I (1955), 100-108. MR0094439 (20:957)

Département de Mathématiques et Statistique, Université Laval, Québec, Canada G1V $0 \mathrm{~A} 6$

E-mail address: patrice.rivard.1@ulaval.ca 